\title{
Cryptogenic fibrosing alveolitis and the fibrosing alveolitis of systemic sclerosis: morphological differences on computed tomographic scans
}

Thomas Y K Chan, David M Hansell, Michael B Rubens, Roland M du Bois, Athol U Wells

\begin{abstract}
Background - The purpose of this study was to identify morphological differences on the computed tomographic (CT) scan between cryptogenic fibrosing alveolitis (CFA) and the fibrosing alveolitis associated with systemic sclerosis (FASSc), and to examine their biological relevance. Methods - One hundred and seven patients with CFA $(n=55)$ or FASSc $(n=52)$ who had undergone thin section $\mathrm{CT}$ scanning were included. Multivariate analysis was used to identify morphological differences on the CT scans between lone CFA and FASSc, and to determine whether the pattern and distribution of disease on the CT scans were functionally significant (as judged by the lung transfer factor (TLCO), forced vital capacity (FVC), and arterial oxygen tension $\left.\left(\mathrm{PaO}_{2}\right)\right)$ or predictive of survival (independent of the type and extent of fibrosing alveolitis, age, sex, and smoking history).
\end{abstract}

Results - Increasingly extensive disease on CT scans was associated with a coarser reticular pattern (increase in reticular score per percentage increase in disease extent $=0.06,95 \%$ confidence interval $(\mathrm{CI})$ 0.03 to $0.09, p<0.0005)$ and increasing upper zone involvement (increase in ratio of upper zone to total disease per percentage increase in disease extent $=0.002$, $95 \%$ CI 0.000 to $0.003, p<0.04)$. Patients with CFA were characterised by a higher upper zone ratio (difference $=0.08,95 \% \mathrm{CI}$ 0.02 to $0.13, p<0.004)$ and a weak trend towards a coarser reticular pattern $(p=$ 0.09), independent of disease extent. Smokers with CFA had more upper zone involvement (difference $=0.11,95 \%$ CI 0.05 to $0.16, p<0.0005)$ and a coarser reticular pattern (difference in reticular score $=$ $1.92,95 \%$ CI 0.27 to $3.55, p<0.02$ ) than smokers with FASSc. The extent of disease on the CT scan was predictive of lung function impairment and survival but the pattern and distribution of disease were not.

Conclusions - Patients with CFA have relatively more upper zone involvement than those with FASSc independent of the extent of disease on the CT scan. This finding may result from smoking related damage but is not functionally significant.

(Thorax 1997;52:265-270)

Keywords: lung fibrosis, scleroderma, CT scanning.

Pulmonary complications are now the most frequent cause of death in patients with systemic sclerosis $(\mathrm{SSc})^{1}$ and pulmonary fibrosis is found at necropsy in over $90 \%$ of cases. ${ }^{2}$ The histopathological features of pulmonary fibrosis associated with systemic sclerosis and cryptogenic fibrosing alveolitis (CFA) are thought to be identical. ${ }^{3}$ On chest radiography, CFA and fibrosing alveolitis associated with systemic sclerosis (FASSc) are generally regarded as indistinguishable. ${ }^{4}$ Furthermore, on high resolution computed tomographic (CT) scanning, which is considered a sensitive means of identifying fibrosing alveolitis, ${ }^{5}$ the features are reported to be similar ${ }^{6}$ and are characterised by a subpleural reticular pattern and small honeycomb cysts. ${ }^{7}$

Despite these apparent similarities, it has been suggested that patients with CFA and FASSc have a different prognosis. In a study of 273 patients with CFA or FASSc, five year survival from the onset of dyspnoea was $50 \%$ in those with CFA and $86 \%$ in those with FASSc. $^{8}$ This marked discrepancy persisted after adjustment for a number of variables including demographic differences, functional indices, and the extent of lung abnormality on the CT scan.

Our impression has been that CFA is characterised by a coarser pattern of fibrosis on CT scanning than FASSc. However, patients with CFA present later in the course of their disease, with more extensive abnormalities on the CT scan. We sought to identify biologically significant differences in the pattern and distribution of disease on the CT scan between CFA and FASSc, after controlling for disease extent.

\section{Methods}

All patients with CFA or FASSc under the care of the Interstitial Lung Disease Unit underwent thin section CT scanning between June 1985 and December 1989. Patients in the following three categories were excluded: (1) those with
19 April 1996
Revised version received
2 October 1996

Accepted for publication

8 October 1996 


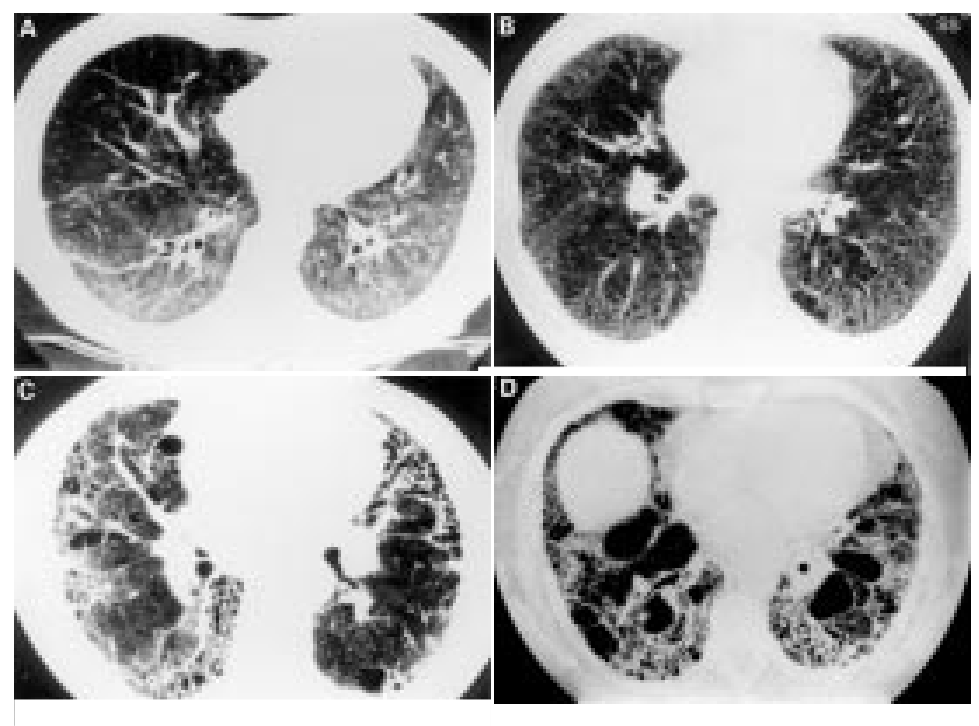

Figure 1 Examples of the four grades of lung disease. (A) Grade 1: fine intralobular fibrosis predominates; (B) grade 2: microcystic pattern with cysts of less than $3 \mathrm{~mm}$ in diameter; (C) grade 3: larger cysts (3-6 mm in diameter) predominate; (D) grade 4: end stage honeycomb lung.
$1 \mathrm{~cm}$ above the dome of the right hemidiaphragm) a visual assessment was made of the extent of abnormal parenchyma to the nearest $5 \%$. An estimate of overall lung involvement was obtained by combining these and applying a weighting factor to correct for reductions in lung volume in the upper zones. This technique of estimating disease extent has been applied to other interstitial lung conditions ${ }^{12}$ and is subject to minor interobserver variation. ${ }^{13}$ The mean of the estimates of the two observers was taken. A ratio of upper zone to total lung involvement was derived by dividing the percentage involvement at the carinal level by the sum at this and the basal level $(1 \mathrm{~cm}$ above the dome of the right hemidiaphragm).

A grading system was used to classify the pattern of lung disease with particular reference to the coarseness of the reticular pattern: ${ }^{4}$ grade $0=\mathrm{a}$ predominantly ground glass pattern; grade 1 = fine intralobular fibrosis predominating; grade $2=$ microcystic pattern with cysts of less than $3 \mathrm{~mm}$ in diameter; grade $3=$ larger cysts (3-6 $\mathrm{mm}$ in diameter); grade $4=$ end stage honeycomb lung (fig 1A-D).

overt emphysema on the CT scan (emphysema has the potential to affect the apparent zonal distribution and pattern of fibrosing alveolitis ${ }^{9}$ ), (2) those with FASSc with obvious end stage pulmonary vascular disease out of proportion to the extent of fibrosing alveolitis (severe pulmonary vascular disease has the potential to affect survival and lung function, independent of lung fibrosis), and (3) those with a predominantly ground glass appearance on the CT scan (this pattern of disease has a different natural history ${ }^{10}$ and can be regarded as a separate clinical entity).

The diagnosis of CFA was made by the presence of (1) persistent bilateral crackles on auscultation, (2) a restrictive defect or isolated depression of total gas transfer on pulmonary function testing, and (3) the absence of other causes of pulmonary fibrosis. Patients with FASSc were included provided that they met the diagnostic criteria of the American Rheumatological Association for Systemic Sclerosis ${ }^{11}$ and had evidence of fibrosing alveolitis by the above criteria.

COMPUTED TOMOGRAPHIC (CT) SCANNING All CT scans were performed on an Elscint 2002 scanner (Elscint, Haifa, Israel) with a scanning time of 5.5 seconds. Sections $3 \mathrm{~mm}$ in thickness were taken at $10 \mathrm{~mm}$ intervals and reconstructed with a high spatial resolution reconstruction algorithm. Sections were obtained at $20 \mathrm{~mm}$ intervals through the lower zones with the patient in the prone position to clarify the effects of gravity in the posterobasal segments of the lower lobes. Window settings were optimised for the lung parenchyma.

All CT sections were examined by two chest radiologists who were not aware of any clinical information. At each of five levels (the origin of the great vessels, mid-arch of the aorta, carina, pulmonary venous confluence, and

\section{CLINICAL INFORMATION}

Demographic details, smoking history, and pulmonary function indices (expressed as percentage predicted) were obtained from the hospital case records. The forced vital capacity (FVC), measured with an Ohio water seal spirometer, the carbon monoxide transfer factor (TLCO), measured by the single breath technique using a PK Morgan respirometer, and arterial oxygen tension in air $\left(\mathrm{PaO}_{2}\right)$ were used in the analysis. Subjects were classified as smokers if they had ever smoked at least one cigarette a day for at least one year. The total intake of cigarettes was also recorded in pack years (excluding six patients without a detailed smoking history). collected from the National Health Central Registrar were examined to obtain survival information to 31 October 1990. In 95 of 107 cases a date of death was established or follow up information was available on or after 31 October 1990.

\section{DATA ANALYSIS}

Results are stated as means with standard deviations. A p value of $<0.05$ was taken to indicate statistical significance. Group comparisons were made using the Student's $t$ test or, when appropriate, non-parametric analysis $\left(\chi^{2}\right.$ statistics or Wilcoxon's rank sum test). Multivariate analyses were performed using STATA software (STATA Computing Resource Center, Santa Monica, USA).

Multivariate models ${ }^{14}$ were used to determine whether the distribution and pattern of disease on the CT scans (examined as dependent variables in separate equations) were independently related to the extent of disease on the scans, the type of fibrosing alveolitis, age, sex, and smoking history. The distribution of disease was evaluated using stepwise forward
Clinical records and/or death certificates 
Table 1 Demographic factors, smoking histories, indices of lung function, frequency of histological confirmation by open lung biopsy and survival in patients with cryptogenic fibrosing alveolitis (CFA) and fibrosing alveolitis associated with systemic sclerosis (FASSc)

\begin{tabular}{llll}
\hline & FASSc $(n=52)$ & CFA $(n=55)$ & p value \\
\hline Age (years) & $49.3(12.4)$ & $61.0(10.6)$ & $<0.00005$ \\
Sex (M:F) & $9: 43$ & $36: 19$ & $<0.00005$ \\
Ever smoked (n) & 17 & 34 & $<0.00005$ \\
TLCO (\% predicted) & $56.0(18.5)$ & $40.6(15.0)$ & $<0.00005$ \\
FVC (\% predicted) & $73.4(21.2)$ & $67.3(20.1)$ & 0.15 \\
$\mathrm{PaO}_{2}$ (kPa) & $11.7(1.5)$ & $9.4(1.9)$ & $<0.00005$ \\
Open lung biopsy (n) $_{\text {Three year survival }}^{35}$ & $95 \%$ & 28 & 0.10 \\
Five year survival & $95 \%$ & $44 \%$ & \\
\hline
\end{tabular}

$\mathrm{TLCO}=$ carbon monoxide transfer factor; $\mathrm{FVC}=$ forced vital capacity; $\mathrm{PaO}_{2}=$ arterial oxygen tension.

Values are mean $(\mathrm{SD})$

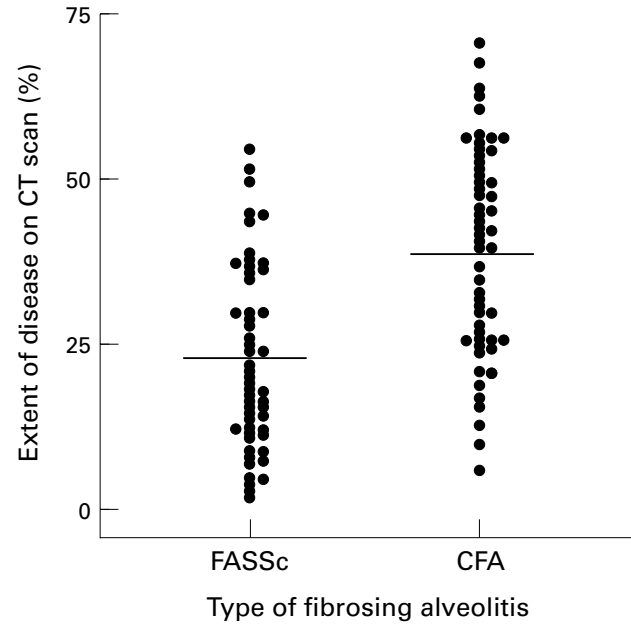

regression; the coarseness of a reticular pattern maximum likelihood ordered logit estimation.

Further stepwise forward regression models were constructed to determine whether depression of FVC, TLCO, and $\mathrm{PaO}_{2}$ (each examined in separate equations) were determined by the pattern and distribution of disease on the CT scans independent of the extent of fibrosing alveolitis, the type of fibrosing alveolitis (CFA versus FASSc), age, sex, and smoking history. In the analysis of survival a proportional hazards model was used to identify the independent determinants of mortality.

\section{Results}

One hundred and forty two patients with a diagnosis of CFA or FASSc underwent CT scanning during the study period. Exclusions consisted of 15 patients with overt emphysema on the CT scan, five patients with FASSc with severe pulmonary vascular disease, 14 patients with a predominant ground glass appearance on the CT scan, and one patient with emphysema and a predominant ground glass appearance, leaving 107 patients eligible for analysis ( 55 with CFA, 52 with FASSc). Prior to death there was lung biopsy evidence of the diagnosis in 28 of the 55 cases of CFA and 35 of the 52 cases of FASSc. Clinical, functional and survival comparisons are presented in table 1 and indicate that patients with CFA were older, more likely to be male, and were characterised by greater functional impairment and a lower survival. Patients with CFA were more likely to have smoked. Smokers with lone CFA had higher pack year histories (median 25, range 1-112) than smokers with FASSc (median 15, range 3-75), $\mathrm{p}<0.04$ (Wilcoxon's rank sum test).

MORPHOLOGICAL DIFFERENCES BETWEEN CFA AND FASSC

Unadjusted differences between CFA and FASSc are shown in figs 2 and 3. The disease was more extensive on the CT scans of patients with CFA than those with FASSc (39.9 (15.8)\% versus $23.0(14.0) \%, \mathrm{p}<0.0001)$. There was proportionally more upper zone involvement (as judged by the ratio of upper zone to total lung involvement) in cases of CFA than FASSc
Figure 2 Extent of fibrosing alveolitis on CT scan, expressed as the percentage of the lungs scored as abnormal, in patients with cryptogenic fibrosing alveolitis (CFA) and fibrosing alveolitis associated with systemic sclerosis (FASSc). Disease was more extensive in CFA $(p<0.0001)$.

(40.4 (8.4)\% versus 30.5 (13.0)\%, p<0.001). There was a greater prevalence of coarse reticular disease (grades 3 and 4 ) in patients with CFA than in those with FASSc $(\mathrm{p}<0.001)$.

On multivariate analysis (table 2), examining the pattern and distribution of disease in separate models, an increasingly coarse reticular pattern was associated strongly with more extensive disease $(p<0.0005)$ and weakly with a diagnosis of CFA $(\mathrm{p}=0.09)$ (equation $R^{2}=$ $0.16)$. Upper zone involvement increased in association with more extensive disease ( $p$ $<0.04)$ and with a diagnosis of CFA $(\mathrm{p}<0.004)$ (equation $\left.R^{2}=0.25\right)$. None of the other covariates (age, sex, smoking history) were independently related to the pattern or distribution of disease on the CT scan. Multi-

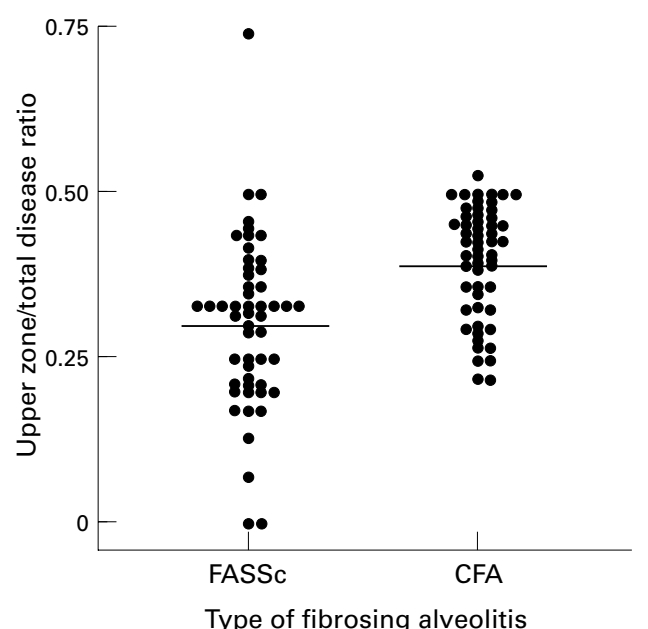

Figure 3 Distribution of fibrosing alveolitis on CT scan, expressed as the ratio of the upper zone in total lung involvement, in patients with cryptogenic fibrosing systemic sclerosis (FASSc). CFA was characterised by relatively more upper zone involvement than FASSc $(p<0.001)$, and this finding persisted after controlling for the global extent of disease in multivariate analysis. alveolitis (CFA) and fibrosing alveolitis associated with 
Table 2 Independent relationships, expressed as regression coefficients with $95 \% \mathrm{CI}$, between CT pattern/distribution and (1) the extent of the disease on the CT scan and (2) the type of fibrosing alveolitis (CFA versus FASSc) in all subjects $(n=107)$, "ever" smokers $(n=51)$, and lifelong non-smokers $(n=56)$

\begin{tabular}{lll}
\hline & Extent of disease on CT scan (1) & Type of fibrosing alveolitis (2) \\
\hline $\begin{array}{l}\text { Coarseness of a reticular } \\
\text { pattern: }\end{array}$ & & \\
All subjects & $0.06(0.03$ to 0.09$)$ & $0.72(-0.18$ to 1.62$)$ \\
"Ever” smokers & $\mathrm{p}<0.0005$ & $\mathrm{p}=0.09$ \\
& $0.08(0.03$ to 0.12$)$ & $1.92(0.27$ to 3.55$)$ \\
& $\mathrm{p}<0.001$ & $\mathrm{p}<0.02$ \\
& $0.05(0.01$ to 0.09$)$ & $0.08(-1.37$ to 1.21$)$ \\
& $\mathrm{p}<0.01$ & $\mathrm{p}=0.91$ \\
& & \\
Non-smokers & $0.002(0.000$ to 0.003$)$ & $0.08(0.02$ to 0.13$)$ \\
Upper zone involvement: & $\mathrm{p}<0.04$ & $0.11(0.05$ to 0.16$)$ \\
All subjects & $0.001(-0.001$ to 0.002$)$ & $\mathrm{p}<0.0005$ \\
"Ever" smokers & $\mathrm{p}=0.41$ & $0.04(-0.03$ to 0.12$)$ \\
& $0.002(0.000$ to 0.008$)$ & $\mathrm{p}=0.28$ \\
\hline
\end{tabular}

Regression coefficients express increases in the reticular score and in upper zone/total disease ratio (1) per unit percentage increase in disease extent and (2) with a diagnosis of CFA.

variate analyses were repeated in 51 "ever" smokers and 56 lifelong non-smokers. In smokers an increasingly coarse reticular pattern was associated with more extensive disease $(\mathrm{p}<0.001)$ and with a diagnosis of CFA $(\mathrm{p}<0.02)$; upper zone involvement increased in association with a diagnosis of CFA $(\mathrm{p}<0.0005)$ but was unrelated to disease extent. By contrast, in non-smokers the coarseness of a reticular pattern and degree of upper zone involvement did not differ independently between patients with CFA or FASSc, but increased with more extensive disease $(p<0.01$ and $\mathrm{p}<0.05$, respectively).

When analyses were repeated, substituting "pack year" smoking histories for "ever/never" smoking histories, the same trends were observed. The coarseness of a reticular pattern was related independently to the extent of disease on the CT scan (increase in reticular score per percentage increase in disease extent $=$ $0.06,95 \%$ confidence interval (CI) 0.03 to $0.09, \mathrm{p}<0.0005)$. Increasing upper zone involvement was seen with more extensive disease (increase in upper zone ratio per percentage increase in disease extent $=0.002,95 \%$ CI 0.001 to $0.004, \mathrm{p}<0.005)$ and in patients with CFA (increase $=0.07,95 \%$ CI 0.01 to 0.12 , $\mathrm{p}<0.04)$.

BIOLOGICAL RELEVANCE OF MORPHOLOGICAL FINDINGS ON CT SCANNING

The severity of functional impairment, as judged by TLCo, FVC, and resting $\mathrm{PaO}_{2}$ levels, was examined in relation to the extent, distribution, and pattern of disease on the CT scan, age, sex, smoking history, and type of fibrosing alveolitis. In separate models, depression of TLCO and FVC, but not $\mathrm{PaO}_{2}$, were positively related to the extent of disease on the CT scan $(\mathrm{p}<0.0005, \mathrm{p}<0.0005$, and $\mathrm{p}=$ 0.13 , respectively). There were no independent relationships between functional indices and the pattern or distribution of disease on the CT scan.

Survival was examined using proportional hazards analysis. A diagnosis of CFA (hazards ratio $12.00,95 \%$ CI 2.38 to $60.3, \mathrm{p}<0.003$ ) and more extensive disease on the CT scan (hazards ratio $1.03,95 \%$ CI 1.00 to 1.07 , $\mathrm{p}<0.04)$ were indicative of poor outcome, but the distribution and pattern of disease were not independently predictive of survival.

\section{Discussion}

The major difference between CFA and FASSc was the relatively greater upper zone involvement on the CT scan in patients with CFA, independent of disease extent on the scan. By contrast, the coarser reticular pattern seen in patients with CFA was strongly associated with more extensive disease on the CT scan and could largely be ascribed to a later clinical presentation. These observations highlight an inherent difficulty in comparing diseases - the possibility that differences are not related to the specific disease but result from differing referral criteria. Patients with CFA were symptomatic at presentation and were referred, in some cases, with progressive disease. Although patients with FASSc were also referred with known or suspected lung fibrosis, the associated systemic disease had served to alert referring physicians to the likelihood of interstitial lung disease. Consequently, it was possible that the observed morphological differences were merely a function of more extensive or more inherently progressive fibrosing alveolitis in a subgroup of patients with CFA referred to our institution, and not true properties of that disease.

The problem of matching populations morphologically can now be overcome in interstitial lung disease. The extent of disease on the CT scan, scored semiquantitatively, has minor observer variation ${ }^{13}$ compared with the considerable observer variation seen in grading histological features. ${ }^{15}$ Moreover, unlike open lung biopsy specimens, CT scanning provides a global assessment of the lung. It also avoids the major drawback of using functional indices to control for disease extent - the confounding influence on lung function of concurrent respiratory disease (such as airflow obstruction or pulmonary vascular disease in FASSc). The use of CT scanning to control for disease extent enabled us to show that the anecdotal observation of a coarser reticular pattern in patients with CFA, confirmed on unadjusted analysis, was no longer statistically significant once overall disease extent was taken into account.

The problem of matching populations for inherent progressiveness of disease, independently of extent, is less straightforward and may be insoluble if diseases differ markedly in their treated course and natural history. In all published studies CFA has been inexorably progressive with a five year survival of only $50 \%$ from the onset of dyspnoea. ${ }^{81617}$ By contrast, despite a histological appearance identical to $\mathrm{CFA},{ }^{3}$ lung fibrosis in systemic sclerosis is often a "hit and run" phenomenon; the prognosis appears to be good except when there is supervening pulmonary vascular disease. A comparison of survival between CFA and FASSc, including the matching of a large number of lung function indices, has established that even 
patients with FASSc with severe functional impairment typical of CFA are not characterised by a CFA-like course. ${ }^{8}$ This conclusion is reinforced by the absence of reports in the literature of patients with progressive FASSc. However, although matching the rate of disease progression may prove to be unattainable, the analysis of survival in the present study provides strong indirect evidence that the greater upper zone involvement in CFA is disease-specific. Had this finding been indicative merely of more progressive disease in both disorders, and not an intrinsic feature of CFA, it should have been independently linked to mortality after adjustment for disease extent in multivariate analysis.

The observation that differences between CFA and FASSc in the pattern and distribution of disease are minor after adjustment for disease extent and irrelevant to functional impairment and survival has important implications for future studies of fibrosing alveolitis. The very different outcome seen in two histologically identical diseases provides an opportunity to gain new pathophysiological insights by identifying differences in lung function indices, bronchoalveolar lavage profiles, and other inflammatory markers which may be central to disease progression. However, an accurate means of controlling for disease extent is indispensable if confounding by severity is to be avoided. ${ }^{18}$ The present study provides strong support for the future use of a simple semiquantitative scoring system of disease extent on the CT scan without the need to control separately for the pattern and distribution. Using this methodology we have recently shown that, for a given disease extent, CFA is characterised by markedly greater arterial hypoxaemia ${ }^{19}$ and higher levels of bronchoalveolar eosinophils ${ }^{20}$ than are seen in FASSc.

In both CFA and FASSc relative increases in upper zone disease were functionally silent. This finding mirrors the observations of Gurney et $a l^{21}$ in a CT study of patients with emphysema; in the erect posture the upper lung zones normally contribute little to resting ventilation and perfusion. The explanation may be less straightforward in fibrosing alveolitis, as the typical lower zone involvement is associated with a marked redistribution of pulmonary blood flow as judged by ventilation perfusion scanning. ${ }^{22}$ However, our findings indicate that relatively greater upper zone involvement is compensated for by relatively less lower zone disease. The absence of an independent relationship between depression of gas transfer and the coarseness of fibrosis suggests that vascular redistribution occurs even in early pulmonary fibrosis; by contrast, cystic lung continues to ventilate, even in advanced fibrosing alveolitis, ${ }^{23}$ accounting for a lack of depression of lung volumes in association with an increasingly coarse reticular pattern.

The present study did not provide a clear explanation for the observed morphological differences between CFA and FASSc, especially the disproportionate upper zone involvement seen in CFA. This finding could be ascribed to smoking, given the vulnerability of the upper lung zones to smoking related damage; morphologic differences between CFA and FASSc were confined to smokers, suggesting that pathogenetic mechanisms in CFA, but not in FASSc, might be modulated by smoking. However, the interpretation of these findings needs to take into account methodological considerations. The use of an "ever/ never" smoker dichotomy places heavy smokers and former smokers with a trivial smoking history in the same category; by contrast, the use of "pack year" smoking histories fails to allow for the fact that the patients most vulnerable to smoking-related damage may be more likely to stop smoking earlier in life, obscuring a dose-related effect. Moreover, if smoking has differing morphological consequences in different diseases, evidence of smoking related damage may fail to achieve statistical significance in a combined patient population (as in our initial multivariate analysis). Finally, the pathogenetic consequences of smoking in established fibrosing alveolitis may depend solely upon cigarette intake during disease progression and may not be unmasked with the use of lifelong smoking histories in analysis. For all these reasons, definitive evidence that smoking influences morphogenesis in fibrosing alveolitis is probably unattainable from non-longitudinal studies which include patients with relatively advanced disease.

In conclusion, patients with CFA are characterised by relatively more upper zone involvement on the CT scan than patients with FASSc. However, this finding is functionally irrelevant and is not directly linked to differences in the rate of progression between the two diseases as judged by survival. The extent of abnormal lung on the CT scan is a suitable means of controlling for disease morphology in future studies of fibrosing alveolitis.

1 Le Roy EC. Sentinel signs and symptoms of systemic sclerosis. Curr Op Rheumatol 1989;1:499-504.

2 Weaver AL, Divertie MB, Titus JL. Pulmonary scleroderma. Dis Chest 1968;54:4-12.

3 Harrison NK, Myers R, Corrin B, Soosay G, Dewar A, Black CM, et al. Structural features of interstitial lung disease in systemic sclerosis. Am Rev Respir Dis 1991;144: 706-13.

4 Fraser RG, Paré JAP, Paré PD, Fraser RS, Genereux GP. Diagnosis of diseases of the chest. 3rd edn. Philadelphia: WB Diagnosis of diseases of the
Saunders, 1988: 1221 .

5 Schurawitzki H, Stiglbauer R, Graninger W, Herold C, Polzleitner D, Burghuber OC, et al. Interstitial lung disease in progressive systemic sclerosis: high resolution CT versus radiography. Radiology 1990;176:755-9.

6 Bergin CJ, Müller NL. CT of interstitial lung disease: a diagnostic approach. AfR 1987;148:815.

7 Müller NL, Miller RR, Webb WR, Evans KG, Ostrow DN. Fibrosing alveolitis: CT-pathologic correlation. Radiology 1986;160:585-8.

8 Wells AU, Cullinan P, Hansell DM, Rubens MB, Black CM, Newman-Taylor A, et al. Fibrosing alveolitis associated with systemic sclerosis has a better prognosis than lone cryptogenic fibrosing alveolitis. Am Rev Respir Dis 1994;149:1583-90.

9 Wiggins J, Strickland B, Turner-Warwick M. Combined cryptogenic fibrosing alveolitis and emphysema: the value of high resolution computed tomography in assessment. Respir Med 1990;84:365-9.

10 Wells AU, Hansell DM, Rubens MB, Cullinan P, Black CM, du Bois RM. The predictive value of appearances CM, du Bois RM. The predictive value of appearances on thin-section computed tomography in fib
veolitis. Am Rev Respir Dis 1993;148:1076-82.

11 Subcommittee for Scleroderma Criteria of the American Rheumatism Association Diagnostic and Therapeutic Criteria Committee. Preliminary criteria for the classification of systemic sclerosis (scleroderma). Arthritis Rheum 1980; 23:581-90.

12 Müller NL, Mawson JB, Mathieson JR, Abboud R, Ostrow $\mathrm{DN}$, Champion N. Sarcoidosis; correlation of extent of disease at CT with clinical, functional and radiographic findings. Radiology 1989;171:613-8. 
13 Collins CD, Wells AU, Hansell DM, Morgan RA, MacSweeney EM, du Bois RM, et al. Observer variation in pattern type and extent of disease in fibrosing alveolitis on thin section computed tomography and chest radiography. Clin Radiol 1994;49:236-40.

14 Hair JF, Andersen RE, Tatham RL, Black WC. Multivariate data analysis with readings. 3rd edn. New York: Macmillan Publishing Company, 1992 .

15 Cherniack RM, Colby TV, Flint A, Thurlbeck WM, Waldron J, Ackerson C, et al. Quantitative assessment of lung pathology in idiopathic pulmonary fibrosis. Am Rev Respir Dis 1991;144:892-900.

16 Turner-Warwick M, Burrows B, Johnson A. Cryptogenic fibrosing alveolitis: clinical features and their influence on fibrosing alveolitis: clinical feature

17 Schwartz DA, Helmers RA, Galvin JR, van Fossen DS, Frees KL, Dayton CS, et al. Determinants of survival in idiopathic pulmonary fibrosis. Am Rev Respir Dis 1994; 149:450-4.

18 Hansell DM, Wells AU. CT evaluation of fibrosing alveolitis: applications and insights. F Thorac Imag 1996;11:231-49.
Wells AU, Hansell DM, Rubens MB, Cailes JB, Black CM, Wells AU, Hansell DM, Rubens MB, Cailes JB, Black CM,
du Bois RM. Functional impairment in lone cryptogenic fibrosing alveolitis and fibrosing alveolitis associated with systemic sclerosis: a comparison. Am $\mathcal{F}$ Respir Crit Car Med 1997 (in press)

20 Wells AU, Hansell DM, Haslam PL, Rubens MB, Cailes J Black CM, et al. Bronchoalveolar lavage cellularity and its relationship to disease extent in fibrosing alveolitis: comparison between the fibrosing alveolitis of systemic
sclerosis and lone cryptogenic fibrosing alveolitis. $A m \mathcal{F}$ Respir Crit Care Med 1996;153:A271.

21 Gurney JW, Jones KK, Robbins RA, Gossman GL, Nelson $\mathrm{KJ}$, Daughton $\mathrm{D}$, et al. Regional distribution of emphysema: correlation of high-resolution CT with emof high-resolution CT with pulmonary function tests in unselected smokers. Radiology

22 Crystal RG, Gadek JE, Ferrans VJ, Fulmer JD, Line BR, Hunninghake GW. Idiopathic pulmonary fibrosis: clinical, histologic, radiographic, physiologic, scintigraphic, cytologic and biologic aspects. Ann Intern Med 1976;85:769 88

23 Strickland NH, Hughes JM, Hart DA, Myers MJ, Lavender JP. Cause of regional ventilation-perfusion mismatching in patients with idiopathic pulmonary fibrosis: a combine CT and scintigraphic study. AfR 1993;160:719-25. 